Sparmannia africana, Lychnis Preslii, Melandryum rubrum; Clematis heterophylla, campaniflora, Aquilegia-, Aconitum-, Trollius-, Coronilla-, Melilotus-, Adenocarpus-, Scorpiurus, Anthyllis-, Orobus-, Lathyrus-, Oxylobium-, Vicia-, Ochrus-, Chorizema-, Rhynchosia-, Clianthus-, Goodia-, Brachysema-, Cytisus-, Astragalus-, Galega-, Halimodendron-, Lotus-, Genista-, Lupinus-, Tetragonolobus-, Trigonella-, Ornithopus-, Bonaveria-, Medicago-, Pisum-, Rolinia-, Baptisia-, Hippocrepis-, Kennedya-, Bossiaea-, Ononis-, Bonjeania-, Lessertia-, Sutherlandia-, Crotalaria-, Edwardsia-, Spartium-Arten; von Ranunculaceen weiter noch einige Ranunculus-, Anemone-, Paeonia-, Hepatica-Arten ; Magnolia-, Helianthemum-, Meconopsis-, Escholtzia-, Chelidonium-, Oxalis-, Mesembryanthemum-species.

Ein Verzeichniss der von mir untersuchten Pflanzenarten, deren Pollen in Wasser sehr schlecht keimt oder gar nicht zur Keimung gelangt, werde ich später veröffentlichen, und bemerke an dieser Stelle noch, dass zu dieser Gruppe die meisten Compositen, Umbelliferen, Malvaceen, Ericaceen, Acanthaceen, Dipsaceen, viele Boraginaceen, Urticaceen, Oucurbitaceen, Verbenaceen, Pedalineen, Plumbagineen, Convolvulaceen, Polemoniaceen, Globulariaceen, Limnantheen, Melastomaceen, Passifloraceen, Simarubaceen, Lythraceen, Celastraceen, 'Tropaeolaceen, Polygalaceen, Onagraceen, Geraniaceen, Rhamnaceen, Malpighiaceen, Ilicineen, Linaceen, Ampelidaceen, Thymeleaceen, Piperaceen, Palmen, Butomaceen, Iridaceen und Juncaceen gehören.

\title{
Bemerkungen über einige orientalische Pflanzenarten.
}

Von Dr. A, von Degen (Budapest).

XXVIII.

\section{Noch ein Wort über Peucedanum obtusifolium Sibth. Sm. (Malabaila obtusifolia Boiss).}

Als ich vor nicht langer Zeit ${ }^{1}$ ) die erste Nachricht über die Wiederauffindung dieser seltenen Pflanze veröffentlichte, geschah dies allein auf Grundlage der von mir an dem asiatischen Ufer des Schwarzen Meeres aufgefundenen Blätter, so dass ich mich jeder Aeusserung über die vermuthliche Gattungszugehörigkeit dieser Art enthalten musste. Diese Blätter, welche genau der Abbildung der Flora Graeca entsprachen ${ }^{2}$ ), aber" so wenig Malabaila-Aehnliches an sich hatten, erregten in mir schon seit Jahren Bedenken, ob denn diese Art von Boissier mit Recht zur Gattung Malabaila gestellt worden sei? Leider konnte ich diesem, ausserdem noch durch das Fragezeichen Nyman's (Consp. p. 287) genährten Zweifel keinen Ausdruck geben, so lange ich die reifen Früchte dieser Pflanze nicht gesehen batte

1) Oesterr. botan. Zeitschr, 1895, p. 67.

2) Sibthorp und Smith, Flora Graeca IIl. tab. 277, p. 70! 
Die Stelle der Beschreibung ${ }^{1}$ ) „fructus ovatus vel obovatus, compresso-planiusculus, obsolete striatus, limbo lato" sprach für Peucedanum, die Fortsetzung aber "limbo ... suberoso, laevi integerrimo, tumidiusculo" konnte mit Boissier auf eine Art der Gattung Malabaila bezogen werden und widersprach der Auffassung De Candolle's, welcher die Pflanze in seinem Prodromus (IV. p. 190) als Pastinaca obstusifolia beschrieben hat.

Die Abbildung der Früchte a. a. O. Jässt uns über die letztgenannte Eigenschaft im Unklaren, und würde die erstere Annahme nur wahrscheinlicher machen, als die für die Gattung Malabaila so charakteristische Form und Zeichnung des Fruchtrandes daran nicht zu erkennen ist. Die fragliche Pflanze ist nun in neuerer Zeit um Constantinopel zu wiederholten Malen gesammelt worden. Herr Nemetz traf sie im blühendem Zustande am asiatischen Ufer; Herr G. A zn avour entdeckte sie schliesslich a ch in Europa, in den Dünen des Schwarzen Meeres zwischen Kila und Domusdere.

Diesem letztgenannten Herrn, einem kritischen Beobachter und vortrefflichen Kenner der Flora Byzantina, verdanke ich die reifen Früchte dieser Doldenpflanze, und die Untersuchung derselben bestätigte meine Vermuthung, dass Sibthorp und Smith die Pflanze ursprünglich richtig beschrieben hatten.

Die Untersuchung der mir nunmehr zur Verfügung stehenden gut ausgereiften Früchte liess keinen Zweifel übrig, dass die von Gattung zu Gattung versetzte Pflanze thatsächlich ein Peucedanum und der Section "Taeniopetala" Vis. (Fl. Dalm. III. p. 49 pro genere) als vorzüglich verschiedene Art neben $P$. urenarium W. K einzureihen sei. In der Form der Früchte ist nämlich die nächste Verwandtschaft mit $P$. arenarium W. K. unverkennbar.

Dieselben sind oval oder eiförmig, an der Basis abgestutzt, flach, jedoch am Rande ebenso wie jene des $P$. arenarium mit einem breiten, etwas dickeren, abgeplatteten Wulste umgeben, "margine latiusculo, crassiusculo, non membranaceo cincta“, wie schon $W$ aldstein und Kitaibel ${ }^{2}$ ) richtig hervorheben; die Dorsalstriemen wie bei den übrigen Taeniopetalen durch dünnes Pericarp gedeckt. Die Früchte des $P$. obtusifolium sind grösser, breiter wie jene des $P$. arenarium und häufig nach der Fläche eingerollt oder eingebogen, ihr Rand ist breiter und dicker wie jener der genannten Art, von welcher sie sich schon durch die ganz andere Form der Blätter auf den ersten Blick unterscheidet.

Während die bisher bekannten Arten dieser kleinen Section untereinander in sehr enger Beziehung stehen ${ }^{3}$ ), stellt $P$. oltusifolium einen von den übrigen weit verschiedenen, durch die eigenthümliche Blattform sehr merkwürdigen 'Typus dar.

Budapest, am 23. December 1896.

1) Flora orientalis II. p. 1054 und schon früher in Ann. d. scienc. nat.

2) Icon. et deser. plant. rar. Hung. I. p. 19.

3) Conf. Oe. B. Z. 1895, p 6i: 11. F. 